\title{
TREMOR: ADAPTAÇÃO DE UMA ESCALA CLÍNICA
}

\author{
DENISE HACK NICARETTA* * JOÃO SANTOS PEREIRA* * MARIA LÚCIA VELUUTINI PIMENTEL **
}

\begin{abstract}
RESUMO - Adaptamos a escala clínica para avaliação de tremor de Fahn, Tolosa e Marín para a monitorização em nosso meio dos pacientes com tremor. Aplicamos essa escala em 123 pacientes com esse distúrbio do movimento, sem restrição etiológica ou de faixa etária, selecionados aleatoriamente no Ambulatório de Medicina Integral e no Serviço de Pronto Atendimento do Hospital Universitário Pedro Ernesto da Universidade do Estado do Rio de Janeiro. Embora tenham sido observadas algumas variáveis influenciando nos escores da escala, estas não parecem ser relevantes para alterar a padronização da mesma e sua aplicabilidade clínica.
\end{abstract}

PALAVRAS-CHAVE: tremor, escala clínica, avaliaçāo.

\section{Tremor: adaptation of a clinical scale}

ABSTRACT - The Fahn, Tolosa e Marín's clinical rating scale for tremor was adapted to our country for evaluation of tremor. This scale was applied to 123 patients with this movement disorder, selected from the Ambulatory of General Medicine at Hospital Universitário Pedro Ernesto from Universidade do Estado do Rio de Janeiro. These patients had been chosen no matter the etiology of their condition or their age. Although some variants may had influenced the scores of this particular scale, they did not seem to change its standardization and applicability.

KEY WORDS: tremor, clinical scale, evaluation.

Tremor é um movimento involuntário rítmico, que pode ser desencadeado pela exacerbaçāo de mecanismos fisiológicos ou ser secundário a enfermidades que afetem o sistema nervoso, como o parkinsonismo, os distúrbios vasculares, as infecções, os tumores, os traumas, as doenças desmielinizantes e as neuropatias periféricas. Dentre estas últimas, destacamos a neuropatia motora e sensitiva hereditária, tipo I e II, as neuropatias secundárias às gamapatias, a polimadiculoneuropatia desmielinizante inflamatória crônica, a neuropatia diabética, a urêmica, a porfirínica e a alcoólica ${ }^{7-}$ ${ }^{10,15-17}$. Pode ainda aparecer isoladamente, como no tremor essencial, ser droga-induzido ou de origem psicogênica ${ }^{7}$. Sua fisiopatologia, até o momento, não foi completamente esclarecida ${ }^{19}$.

Segundo a sua atitude motora, $o$ tremor pode ser assim classificado $o^{7,12}$ :

1. Tremor de repouso - grosseiro, manifesta-se na ausência de atividade motora voluntária. A parte corporal onde o tremor se apresenta deve estar completamente apoiada, de maneira a não sofrer a ação da gravidade. Seu maior exemplo é o tremor parkinsoniano.

2. Tremor de ação - ocorre com a contraçāo voluntária da musculatura durante o movimento. Inclui o tremor postural e o cinético.

Ambulatório de Distúrbios do Movimento, Disciplina de Neurologia do Hospital Universitário Pedro Ernesto (HUPE) da Universidade do Estado do Rio de Janeiro (UERJ): *Bolsista CNPq; **Professor Adjunto da Disciplina de Neurologia da UERJ, responsável pelo Ambulatório de Distúrbios do Movimento; ***Médica neurologista. Apoio CNPq. Aceite: 7-abril-1997.

Dr. Joāo Santos Pereira - Disciplina de Neurologia, Hospital Universitário Pedro Ernesto, UERJ - Boulevard 28 de Setembro 77, $2^{\circ}$ andar - 20551-030 Rio de Janeiro RJ - Brasil. 
2.1. Tremor postural - aparece com a manutenção de uma posição voluntária contra a ação da gravidade, como quando os membros superiores estão estendidos à frente do corpo, cm postura antigravitacional. Podemos identificá-lo no tremor fisiológico, no fisiológico exagerado, no essencial, no droga-induzido, no tremor em adejar e ainda na doença de Parkinson (tremor postural da doença de Parkinson).

2.2. Tremor cinético - aparece com qualquer movimento, podendo ocorrer durante o seu início, no seu decorrer ou na sua finalização, sendo respectivamente denominado de inicial, de transição e terminal. Tende a aumentar a sua amplitude quanto mais próximo ao alvo. Segundo Findley ${ }^{7}$, o termo intencional tem sido utilizado para definir o tremor desencadeado durante a realização de um movimento preciso, ausente em repouso e na primeira fase do movimento voluntário; é evidenciado melhor no final do movimento, quando de encontro ao alvo. É visto nas afecções cerebelares, em especial na esclerose múltipla; e também nas lesões do tronco cerebral, e ainda em algumas apresentações do tremor essencial?

Embora essas características possam estar todas presentes em um mesmo individuo, sempre haverá o predomínio de uma dessas ${ }^{10,19}$.

A avaliação, por métodos complementares, desse distúrbio do movimento ainda não é satisfatória. Para Bain e col. ${ }^{1}$ as escalas clínicas, para acompanhamento e avaliação da eficácia terapêutica, seriam mais válidas que a técnica padronizada de acelerometria convencional. Assim Fahn, Tolosa e Marín ${ }^{5.6}$ optaram por desenvolver uma escala clínica que quantificasse não só o tremor postural, mas também o de repouso, o de açāo/intençāo e o de voz. Essa escala avaliaria também o impacto do tremor na habilidade do paciente em manter suas atividades diárias, e em outras tarefas específicas das mãos, relacionadas, por exemplo, aos cuidados higiênicos e com o vestir.

Traduzimos e adaptamos essa escala, para o nosso meio, na tentativa de torná-la um instrumento simples e padronizado de apreciação e monitorização clínica e terapêutica dos pacientes com tremor, de qualquer etiologia.

\section{PACIENTES E MÉTODOS}

A escala clínica para avaliação de tremor de Fahn, Tolosa e Marín foi aplicada, sempre pelo mesmo neurologista, nos pacientes que apresentavam tremor (sem restrição etiológica ou de faixa etária), atendidos no Serviço de Pronto Atendimento e no Ambulatório de Medicina Integral do Hospital Universitário Pedro Ernesto da Universidade do Estado do Rio de Janeiro, no periodo de maio-1995 a janeiro-1996. Foram selecionados aleatoriamente 123 pacientes.

Essa escala foi traduzida para o nosso idioma, assim como os parâmetros para graduação dos achados. É dividida em três partes, nas quais avalia, respectivamente, a localização e a gravidade do tremor, tarefas motoras cspecíficas e o impacto do tremor sobre as atividades diárias do paciente. Sua aplicação e o processamento dos resultados estavam $\mathrm{em}$ acordo com as especificações dos referidos autores.

Todos os pacientes examinados foram esclarecidos dos propósitos e objetivos deste estudo, antes do inicio dele, dando seu consentimento por escrito.

\section{RESULTADOS}

Os pacientes estudados, segundo as características clínicas do tremor, apresentavam tremor fisiológico exacerbado, tremor essencial, tremor essencial familiar e tremor parkinsoniano. Trinta e seis pacientes nunca haviam notado este distúrbio do movimento previamente à aplicaçāo da escala.

Trinta e oito pacientes pertenciam ao sexo masculino $(30,89 \%)$ e 85 ao feminino $(69,11 \%)$. Vinte e dois eram mestiços (18,03\%), 32 negros (26,23\%), e 68 brancos (55,74\%). A média de idade dos pacientes foi 51 anos, sendo a idade mínima 11 anos e a máxima 84 anos.

Dos 123 pacientes avaliados, o maior escore alcançado foi $34,62 \%$ e o menor $3,20 \%$. Já quando separados por sexo, o maior escore para o masculino foi $34,62 \%$ e o menor $5,77 \%$, e para o feminino $22,44 \%$ e $3,20 \%$ respectivamente. 
Tabela 1. Demonstração do grau de prejuizo funcional atribuído pelo paciente e pelo examinador.

\begin{tabular}{cccccc}
\hline Pontuação & 0 & 1 & 2 & 3 & 4 \\
\hline grau de prejuízo funcional: cxaminador & 91 pacientes & 4 pacientes & 1 paciente & - & - \\
grau de prejuízo funcional: paciente & $\mathbf{8 2}$ pacientes & $\mathbf{8}$ pacientes & 1 paciente & $\mathbf{3}$ pacientes & - \\
\hline
\end{tabular}

Na avaliação realizada pelo paciente, quanto ao grau de prejuízo funcional determinado pelo seu tremor, apenas 96 destes foram capazes de atribuir um valor que variava de 0 a 4 , conforme demonstrado na Tabela 1. Vinte e sete não o fizeram, por não conseguirem entender o propósito da questão formulada, a despeito da clareza com que foi exposta a pergunta.

Para comparação dos escores segundo faixa etária foi utilizado o teste não paramétrico KruskalWallis com $\mathrm{H}$ de 16,97 . Houve diferença significativa $(\mathrm{p}<0,01)$. Para avaliarmos esta diferença foi aplicada a comparação múltipla com dms de $5 \%$ com valor igual a 93,43 . Assim, para as faixas etárias entre 10 e 50 anos houve comportamento semelhante em relação ao escore, enquanto que, nas faixas etárias a partir dos 51 anos, notou-se aumento do escore proporcional a faixa etária $(\mathrm{p}<0,05)$.

Foi utilizado o coeficiente de Spearman para verificar a dependência da idade em relação ao escore; através dos dados aferidos observamos que, com aumento da idade, aumentava o escore ( $p<$ 0,$01 ; r=0,32$ ).

Quando comparamos o escore segundo o sexo, aplicamos o teste não paramétrico de MannWhitney $\left(U^{*}=2,28\right)$ e verificamos que existia diferença significativa $(p<0,01)$ em relação ao sexo masculino, no qual predominaram os maiores escores. a Tabela 2 .

Em relação à escolaridade dos pacientes, 10 eram analfabetos, e os demais distribuídos segundo

Setenta e dois pacientes encontravam-se em pleno exercício de suas atividades profissionais. Dezesseis deles eram aposentados e 11 estudantes. Vinte e quatro pacientes, pertencentes ao sexo feminino, não exerciam atividade profissional.

Vinte e três pacientes eram tabagistas e 10 etilistas. Noventa e cinco pacientes ingeriram bebidas que continham cafeína, em um período de até 8 horas antes da avaliação. Nenhum havia ingerido bebidas alcóolicas.

Tabela 2. Distribuição por escolaridade e faixa etária dos pacientes.

\begin{tabular}{ccccccc}
\hline Faixa etária & $\begin{array}{c}1^{\circ} \mathrm{grau} \\
\text { completo }\end{array}$ & $\begin{array}{c}1^{\circ} \text { grau } \\
\text { incompleto }\end{array}$ & $\begin{array}{c}2^{\circ} \text { grau } \\
\text { completo }\end{array}$ & $\begin{array}{c}2^{\circ} \text { grau } \\
\text { incompleto }\end{array}$ & $\begin{array}{c}3^{\circ} \text { grau } \\
\text { completo }\end{array}$ & $\begin{array}{c}3^{\circ} \text { grau } \\
\text { incompleto }\end{array}$ \\
\hline $0-20$ & 2 & 5 & 1 & 3 & - & 7 \\
$21-30$ & - & 1 & - & 2 & - & - \\
$31-40$ & 1 & 10 & 1 & 2 & 1 & - \\
$41-50$ & 2 & 17 & 1 & - & - & - \\
$51-60$ & 1 & 17 & - & 1 & 1 & - \\
$61-70$ & - & 18 & - & - & - & - \\
$71-80$ & - & 15 & - & - & - & - \\
$81-90$ & - & 4 & - & - & - & - \\
Total & 6 & 87 & 3 & 8 & 2 & 7 \\
\hline
\end{tabular}


Tabela 3. Localização do tremor pelos sítios anatômicos.

\begin{tabular}{cc}
\hline Sítio anatômico & Número de pacientes \\
\hline face & 4 \\
língua & 6 \\
cabeça & 10 \\
membro inferior direito & 16 \\
membro inferior esquerdo & 19 \\
membro superior esquerdo & 115 \\
membro superior direito & 117 \\
\hline
\end{tabular}

Cento e quinze pacientes eram destros, 7 sinistros e 1 ambidestro. Noventa e dois pacientes tiveram a mão direita mais acometida e 25 , a esquerda. Seis não tiveram predominância de acometimento.

O tremor distribuiu-se pelos sítios anatômicos mostrados na Tabela 3, podendo cada paciente apresentar mais de um segmento acometido. Nenhum paciente apresentou tremor de voz, de tronco e ortostático.

O tempo gasto para aplicação da escala variou de 20 a 50 minutos.

\section{DISCUSSÃO}

Dentre os métodos complementares que dispomos para avaliação do tremor está o acelerômetro, que requer um laboratório e instrumentação especializada, e raramente é acessível a todos neurologistas ${ }^{5,6}$. Além disto, este método não avalia o tremor postural de membros superiores'. Já os aparelhos de deteç̧ão ou interrupção de luz, como os goniômetros de luz polarizada são de difícil obtenção. Por sua vez, a gravação em videotape não permite a percepção de modificações discretas, ou mesmo a qualificação adequada do tremor ${ }^{\mathrm{s}, \mathrm{h}}$, pois quanto maior a frequência deste, menor a sensibilidade da película em registrá-lo'.

Ocorre confusão diagnóstica quanto à classificaçāo do tremor, principalmente quando este tem múltiplas origens, e apresenta-se concomitantemente a outras desordens do movimento, ou ainda é sobreposto ao tremor fisiológico exacerbado. Uma das dificuldades em se analisar o tremor reside no fato de que existe pouco ou nenhum consenso quanto ao método mais apropriado para quantificá-lo, além de existirem numerosas variáveis que devem ser considcradas, dentre clas, a avaliação em diferentes articulações, em diferentes posturas, em diferentes frequências, bem como diferentes instruções para o indivíduo examinado. Assim, esta falta de padronização dificulta ao próprio investigador interpretar os dados obtidos. Frequentemente os resultados podem não ser deduzidos de modo adequado, ou podem mesmo parecer contraditórios, em especial se considerarmos a amplitude do tremor ${ }^{3}$.

Nestas últimas décadas, com a evolução da eletrônica, muitos métodos quantitativos para mensurar o tremor foram desenvolvidos, mas nenhum é ainda adequado. Não raro são complementados com escalas clínicas. Estas, por sua vez, são criticadas por serem imprecisas e subjetivas, e não detectarem, por conseguinte, pequenas mudanças na amplitude do tremor ${ }^{4}$. Outrossim, nenhuma das escalas clínicas teve aceitação ou validação universal. A vantagem da escala clínica sobre o acelerômetro baseia-se no fato de que é mais viável economicamente, e de aplicação mais fácil. Sua compreensão fica muito mais acessível para a maioria dos médicos, e até mesmo para os proprios pacientes. Para propósito de avaliação de ensaios clínicos a escala produz resultados confiáveis ${ }^{1}$. 
Sprague e col. ${ }^{18}$ referiram que, embora um grande número de esquemas para avaliação do tipo e proporção da discinesia tardia tenham sido utilizados, poucas tentativas ocorreram para se atualizar escal as que dependessem estritamente da observação humana. Para esses autores, os métodos técnicos são complementados com vantagens por escalas clínicas. No entanto, não afastaram a necessidade de estudos cruzados para se estabelecer as verdadeiras vantagens da interrelação entre escalas clínicas e técnicas instrumento dependentes. Lejoyeux e col." traduziram a escala de Simpson e Angus para avaliação dos sintomas extrapiramidais, concluindo ser essa de uso fácil e não despender muito tempo para sua aplicaçăo.

Não devemos esquecer, no entanto, que utilizar escalas de diferentes autores, para avaliar uma mesma afecção representa um problema na interpretaçāo e comparaçāo dos resultados ${ }^{14}$. Essas escalas podem ser satisfatórias para a pesquisa clínica mas, ao nível experimental, tornam-se muito subjetivas ${ }^{4}$,como já referido anteriormente.

A condição essencial para que uma escala possa aferir determinado fenômeno reside na sua capacidade de avaliar igualmente tanto a piora dos pacientes, como os extremos de resultado dessa mesma população ${ }^{13}$. A observação do próprio paciente é muito importante, devendo-se portanto acrescentar a qualquer protocolo os questionários de autoavaliação, principalmente se levarmos em conta que no tremor essencial, por exemplo, o prejuízo funcional, ou a incapacidade do paciente na realização de suas atividades manuais, nāo se correlacionam com a amplitude do tremor².

Fahn e col. ${ }^{3.6}$ elaboraram uma escala que pudesse suprir todas essas falhas e padronizar a aplicação e observação do mesmo fenômeno, de modo a ser utilizada por diferentes examinadores, em diferentes centros, sem prejuízo na qualidade da observação. Padronizaram-se condições e definiçōes para a intensidade do tremor, para comparação de uma avaliação com as anteriores ou subsequentes, ou mesmo enire examinadores. Nessa escala clínica nenhum instrumento especial é necessário, podendo ser utilizada para avaliar tremor de diferentes etiologias. Tanto a gravidade do tremor quanto seu impacto sobre a atividade diária do paciente são avaliadas, permitindo que paciente e examinador possam quantificar, objetivamente, este prejuízo funcional ${ }^{5,6}$.

Notou-se apreensão por parte dos pacientes, por nós examinados, em relação à avaliação, apesar de esclarecidos que não se tratava de teste de performance intelectual. A ansiedade, independente da idade, sexo, cor ou nível sócio-cultural, pode ter sido provocada pela própria consulta médica, e talvez agravada pela investigaçāo ncurológica, fato para o qual deveremos estar atentos quando da interpretação dos resultados.

O fato de $8,13 \%$ dos pacientes serem analfabetos não comprometeu a análise, pois estes foram plenamente capazes de copiar a frase padrão.

A aplicação da escala se mostrou extremamente variável em relaçāo ao tempo gasto, que foi dependente do desembaraço do médico e do paciente em realizar as tarefas propostas. Esse aspecto deve ser considerado caso essa escala seja utilizada em locais com finalidade estritamente assistencial.

Embora sejam observadas algumas variáveis que influenciam os escores da escala, estas não parecem ser relevantes para alterar a padronização da mesma e sua aplicabilidade clínica.

Agradecimentos - Aos médicos do Serviço de Pronto Atendimento e do Ambulatório de Medicina Integral do Hospital Universitário Pedro Emesto da Universidade do Estado do Rio de Janeiro.

\section{REFERÊNCIAS}

1. Bain PG, Findley $L$, Atchison P, Behari $M$, Vidailhet $M$, Gresty M, Rothwell JC, Thompson PD, Marsden CD. Assessing tremor severity. J Neurol Neurosurg Psychiatry 1993:56:868-873.

2. Bain PG. Mally J, Gresty M. Findley LJ. Assessing the impact of essential tremor on upper limb function. J Neurol 1993;241:54-61.

3. Beuter A, Geoffroy A, Cordo P. The measurement of tremor using simple laser systems. J Neuros Meth 1994;53:47-54. 
4. Elble RJ, Koller WC. The measurement and quantification of tremor. In: Elble RJ, Koller WC. Tremor. Baltimore: John Hopkins Univ Press, 1990:10-36.

5. Fahn S, Tolosa E, Marín C. Clinical rating scale for tremor. In: Jankovic J, Tolosa E (eds). Parkinson's disease and movement disorders. Baltimore: Urban \& Schwarzenberg, 1988:225-234.

6. Fahn S, Tolosa E, Marin C. Clinical rating scale for tremor. In: Jankovic J, Tolosa E (eds). Parkinson's disease and movement disorders. Ed2. Baltimore: Urban \& Schwarzenberg, 1993:271-280.

7. Findley LJ. Tremors: differential diagnosis and pharmacology. In Jankovic J, Tolosa E (eds). Parkinson's disease and movement disorders. Baltimore: Urban \& Schwarzenber, 1993;293-313.

8. Freitas MRG. Doença de Charcot-Marie-Tooth: estudo de 45 pacientes. Tese (Professor Titular) Universidade Federal Fluminense. Niterói, 1993.

9. Koller WC, Biary N, Cone S. Disability in essential tremor: effect of treatment. Neurology 1986;36:1001-1004.

10. Koller WC, Huber SJ. Tremor disorders of aging: diagnosis and management. Geriatries 1989;44:33-41.

11. Lejoyeux M, Gorwood P, Stalla-Bourdillon A, Adès J. Traduction et utilisation de l'échelle de Simpson et Angus de symptomes extra-pyramidaux. Encéphale 1993;19:17-21.

12. Lou J-S, Jankovic J. Tremors. In Appel SH (ed). Current Neurology. St Louis: Mosby Year Book, 1991:199-232.

13. MacKenzie RC, Charlson ME. Standards for the use of ordinal scales in clinical trials. Br Med J 1986;292:40-43.

14. Martínez-Martín P, Gil-Nagel A, Gracia LM, Gómez JB, Martínez-Sarriés J, Bermejo F, The Cooperative Multicentric Group. Unified Parkinson's disease rating scale characteristics and structure. Mov Disord 1994;9:76-83.

15. Nascimento OJM. Polirradiculoneuropatia desmielinizante inflamatória crônica (Síndrome de Austin-Dyck): estudo de 45 pacientes. Tese (Professor Titular) Universidade Federal Fluminense. Niterói, 1993.

16. Nascimento OJM, Freitas MRG, Chimelli L, Nevares MT, Escada TM. Polineuropatias das gamapatias monoclonais de etiologia indeterminada. Rev Bras Neurol 1993;29:140-146.

17. Said G, Bathien N, Cesaro P. Peripheral neuropathies and tremor. Neurology 1982;32:480-485.

18. Sprague RL, Korach MS, Van Emmerik REA, Newell KM. Correlations between kinematic and rating scale measures of tardive dyskinesia in a developmentally disabled population. J Nerv Ment Dis 1993;181:42-47.

19. Weiner WJ, Lang AE. Tremor. In: Weiner WJ, Lang AE. Movement disorders: a comprehensive survey. New York: Futura 1989:221-256. 